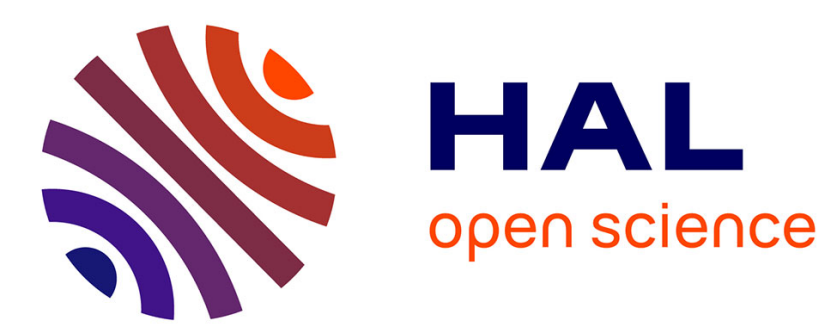

\title{
An introgression breakthrough left by an anthropogenic contact between two ascidians
}

\author{
Alan Le Moan, Charlotte Roby, Christelle Fraisse, Claire Daguin-Thiébaut, \\ Nicolas Bierne, Frédérique Viard
}

\section{- To cite this version:}

Alan Le Moan, Charlotte Roby, Christelle Fraisse, Claire Daguin-Thiébaut, Nicolas Bierne, et al.. An introgression breakthrough left by an anthropogenic contact between two ascidians. Molecular Ecology, inPress, 10.1111/mec.16189 . hal-03355754

\section{HAL Id: hal-03355754 https://hal.science/hal-03355754}

Submitted on 27 Sep 2021

HAL is a multi-disciplinary open access archive for the deposit and dissemination of scientific research documents, whether they are published or not. The documents may come from teaching and research institutions in France or abroad, or from public or private research centers.
L'archive ouverte pluridisciplinaire HAL, est destinée au dépôt et à la diffusion de documents scientifiques de niveau recherche, publiés ou non, émanant des établissements d'enseignement et de recherche français ou étrangers, des laboratoires publics ou privés. 
1 Accepted in Molecular Ecology (Sept. 2021)

2 Available on BioRxiv: doi: https://doi.org/10.1101/2021.08.05.455260

3

4

5 An introgression breakthrough left by an anthropogenic contact between two

6 ascidians

7 Alan Le Moan ${ }^{1,2,}{ }^{*}$, Charlotte Roby ${ }^{1}$, Christelle Fraisse ${ }^{3}$, Claire Daguin-Thiébaut ${ }^{1}$, Nicolas

8 Bierne $^{4}$, Frédérique Viard ${ }^{1,4, *}$

$9{ }^{1}$ Sorbonne Université, CNRS, UMR 7144, Station Biologique de Roscoff, Place Georges Teissier,

1029680 Roscoff, France

112 Department of Marine Sciences, Tjärnö Marine Laboratory, University of Gothenburg,

12 Laboratorievägen 10, 45296 Strömstad, Sweden

$13{ }^{3}$ CNRS, Univ. Lille, UMR 8198 - Evo-Eco-Paleo, F-59000 Lille, France

$14{ }^{4}$ ISEM, Univ. Montpellier, CNRS, EPHE, IRD, Montpellier, France

15 *corresponding authors: alan.le.moan@gmail.com, frederique.viard@umontpellier.fr

16

17 Running title

18 Introgression footprint of a sea squirt invasion 


\section{Abstract}

20 Human-driven translocations of species have diverse evolutionary consequences such as

21 promoting hybridization between previously geographically isolated taxa. This is well-

22 illustrated by the solitary tunicate, Ciona robusta, native to the North East Pacific and

23 introduced in the North East Atlantic. It is now co-occurring with its congener $C$. intestinalis in

24 the English Channel, and C. roulei in the Mediterranean Sea. Despite their long allopatric

25 divergence, first and second generation crosses showed a high hybridization success between

26 the introduced and native taxa in the laboratory. However, previous genetic studies failed to

27 provide evidence of recent hybridization between $C$. robusta and $C$. intestinalis in the wild.

28 Using SNPs obtained from ddRAD-sequencing of 397 individuals from 26 populations, we

29 further explored the genome-wide population structure of the native Ciona taxa. We first

30 confirmed results documented in previous studies, notably i) a chaotic genetic structure at

31 regional scale, and ii) a high genetic similarity between $C$. roulei and $C$. intestinalis, which is

32 calling for further taxonomic investigation. More importantly, and unexpectedly, we also

33 observed a genomic hotspot of long introgressed $C$. robusta tracts into $C$. intestinalis genomes

34 at several locations of their contact zone. Both the genomic architecture of introgression,

35 restricted to a $1.5 \mathrm{Mb}$ region of chromosome 5 , and its absence in allopatric populations

36 suggest introgression is recent and occurred after the introduction of the non-indigenous

37 species. Overall, our study shows that anthropogenic hybridization can be effective in

38 promoting introgression breakthroughs between species at a late stage of the speciation

39 continuum.

40 Keywords: Biological introductions, non-indigenous species, anthropogenic hybridization,

41 introgression hotspots, population genomics, tunicates 


\section{Introduction}

Anthropogenic hybridizations can arise from human-mediated translocations of

44 species outside their natural distribution range, which promote secondary contact between

45 previously geographically isolated taxa (McFarlane \& Pemberton, 2019). Such circumstances

46 enable the initial phase of the hybridization to be studied in a natural context (Grabenstein \&

47 Taylor, 2018, Hufbauer et al., 2012, Faust, Halvorsen, Andersen, Knutsen, \& André, 2018;

48 Popovic, Matias, Bierne, \& Riginos, 2020). Anthropogenic hybridizations thus provide unique

49 opportunities to examine gene flow between species experiencing incomplete reproductive

50 isolation, even at a late stage of the speciation process (Viard, Riginos, \& Bierne, 2020).

Species introductions are common and occur at increasing rates in the marine

52 realm (Seebens et al., 2017). Ports and marinas, one component of the increasing marine

53 urbanization, are one point-of-entry of many non-native species (Firth et al., 2016), where

54 they can co-occur with native congeners (e.g., Bouchemousse, Lévêque, Dubois, \& Viard,

55 2016b). Consequently, these habitats are prone to facilitating hybridization between

56 introduced and native species. They provide a suitable system to examine secondary gene

57 flow in case of anthropogenic hybridization. This is illustrated by a recent study from Simon et

58 al. (2020), which documented the presence of a singular lineage, named "dock mussels",

59 originating from a recent admixture between Mytilus edulis, native to the North Atlantic, and

60 Mytilus galloprovincialis, native to the Mediterranean Sea. These admixed populations are

61 restricted to port habitats in European waters.

The secondary contact between the solitary tunicates Ciona robusta and Ciona

63 intestinalis in the English Channel is another case study, but with very different outcomes.

64 Ciona robusta (formerly known as C. intestinalis type A; Gissi et al., 2017), native to Asia, was 
65 introduced in the early 2000s to the English Channel (Bouchemousse, Bishop, \& Viard, 2016a),

66 the native range of $C$. intestinalis (formerly known as C. intestinalis type B; Gissi et al., 2017).

67 The two species are found in syntopy (Nydam \& Harrison, 2010, Bouchemousse et al., 2016b),

68 display similar life-cycles (Bouchemousse, Lévêque, \& Viard, 2017), and can be easily crossed

69 in the laboratory (Bouchemousse et al., 2016b; Malfant, Coudret, Le Merdy, \& Viard, 2017)

70 despite their high molecular divergence $(12 \%$ of net synonymous divergence, Roux,

71 Tsagkogeorga, Bierne, \& Galtier, 2013, Roux et al., 2016). Successful hybridization nonetheless

72 occurs in one direction only, with C. intestinalis as the maternal lineage (Bouchemousse et al.,

73 2016b; Malfant, Darras, \& Viard, 2018). Demographic inferences based on few individuals but

74 high number of markers derived from 852 coding sequences (total length of $270 \mathrm{~kb}$ ) suggested

75 the presence of several introgression hotspots between the two species (Roux et al., 2013).

76 However, by using 100 ancestry-informative SNPs, this introgression was later shown to be

77 the outcome of past introgression, far preceding the contemporary secondary contact

78 nowadays observed in the English Channel (Bouchemousse, Liautard-Haag, Bierne, \& Viard,

79 2016c). In Europe, where both species occur in sympatry, Bouchemousse et al. (2016c) have

80 found limited evidence for hybridization (i.e., one F1 hybrid out of 449 individuals), and no

81 sign of contemporary introgression (i.e., no F2s or backcrosses). Thus, despite a high

82 hybridization potential, efficient reproductive barriers seem to prevent hybridization in the

83 wild between the native and non-native species. Although these results are based on low

84 genomic coverage, they suggest that introgression between $C$. intestinalis and C. robusta is far

85 less common than in Mytilus species. Indeed, admixture was effectively detected in dock

86 mussels using similar genomic coverage (Simon et al., 2020). Nevertheless, high genomic

87 coverage can reveal subtler introgression patterns as exemplified in model systems in 
88 speciation (sticklebacks: Ravinet et al., 2018; rock periwinkle: Stankowski et al., 2020; drosophila: Turissini \& Matute, 2017), as well as in native-invasive systems (cotton bollworm:

90 Valencia-Montoya et al., 2020; honey bee: Calfee, Agra, Palacio, Ramírez, \& Coop, 2020). In this study, we further explored the genome-wide population structure of the native tunicate $C$. intestinalis in the North Atlantic using a large number of SNPs provided by a ddRAD-sequencing approach. Our study expands upon the work conducted by Hudson et al.

94 (2020), which described multiple glacial lineages of $C$. instestinalis within Europe. Here, we 95 aim to evaluate the consequences of anthropogenic hybridization with its congener $C$. robusta that has been introduced in the range of one $C$. intestinalis glacial lineage, in the English Channel. As a control, and for the sake of comparison, we also examined one population of the native species Ciona roulei from the Mediterranean Sea. The species status of $C$. roulei has been repeatedly questioned (Lambert, Lafargue, \& Lambert, 1990; Nydam \& Harrison, 2010; Malfant et al., 2018), and it might better be described as an isolated population of $C$. intestinalis. Interestingly, $C$. roulei can be found in sympatry with $C$. robusta, also introduced in the Mediterranean Sea. Based on genome-wide SNPs, we 1. recover the population structure described from previous studies for $C$. intestinalis both at fine and large geographical scales 2. provide genome-wide support for a revision of the taxonomic status of $C$. roulei, and 3. provide the first evidence in favor of recent introgression events from $C$. robusta towards C. intestinalis in their contact zone, but not in allopatric populations. However, introgression is restricted to a $1.5 \mathrm{Mb}$ region of chromosome 5 . Overall, our study shows that anthropogenic 108 hybridization can be effective in promoting gene flow even between species at a late stage of speciation, but in this case introgression can be restricted to localized breakthroughs in the 110 receiving genome. 
112 Materials and methods

113 Sample collection

We studied 397 individuals of Ciona spp., previously sampled across the North

115 Atlantic. The sampling locations are shown in Figures 1B (fine-scale) and 2A (large-scale) with 116 details provided in Table S1 in the Supporting Information file. Most individuals $(\mathrm{N}=346)$ were 117 C. intestinalis sampled from 22 locations in 2012 by Bouchemousse et al. (2016a), except for 118 one site (Jer) that was sampled in 2014 by Hudson, Viard, Roby, \& Rius (2016). This sampling 119 includes two locations (REK, Iceland, and NAH, US) where C. intestinalis is most likely 120 introduced although its status remains debated (i.e., cryptogenic; See Appendix 1 in 121 Bouchemousse et al., 2016a). The sampling scheme aims at covering the known geographic 122 range of this species, with a focus on the English Channel where the species coexists with its 123 introduced congener $C$. robusta. In addition, 19 specimens of $C$. roulei, native to the 124 Mediterranean Sea, were included, along with 32 individuals from the introduced species $C$. 125 robusta, of which 16 were sampled from the Mediterranean Sea (in sympatry with C. roulei) 126 and 16 from the English Channel (in sympatry with $C$. intestinalis).

\section{DNA extraction and library preparation}

129 to the manufacturer's protocol (Macherey-Nagel, Germany). Individual double-digest RAD130 seq libraries were constructed according to a protocol slightly modified from Brelsford, 131 Dufresnes, \& Perrin (2016). A detailed step-by-step protocol is available at 132 dx.doi.org/10.17504/protocols.io.bv4tn8wn. Briefly, DNA was digested with Pstl and Msel 133 after fluorometric quantification of DNA concentration with PicoGreen (Invitrogen, Carlsbad, 
CA, USA) and normalization of the extracts. Each individual was labelled with a unique barcode-index combination, with an inline barcode (incorporated in the Pstl adaptor) and an

136 Illumina Truseq index (incorporated during the PCR carried out on the ligation products). Size 137 selection was carried out with $1.5 \%$ agarose cassettes in a pippin prep (Sage Science) to select 138 fragments between 280 and 600 base pairs. A total of three pooled libraries were sequenced, 139 each containing 184 individuals, with replicates (two individuals per library and two across the 140 three libraries). Each library was sequenced in two lanes of an Illumina HiSeq 2500 v4 high 141 throughput flow cell generating 125 base single-end reads at Eurofins Genomics (Ebersberg, 142 Germany).

\section{Bioinformatics pipeline}

The reads were demultiplexed based on their individual index-barcode with the processRADtags programme of Stacks v2 (Rochette, Rivera- Colón, \& Catchen, 2019). Reads with ambiguous barcodes or low sequencing quality were removed. Overall, an average of

$1471.7 \mathrm{M}$ good quality reads per sample was kept for the downstream analyses. The reads were 148 trimmed to 80 base pairs, and mapped on the C. robusta genome (KH79 version, Dehal et al., 2002, NCBI assembly GCF_000224145.1) using the default parameters implemented in BWA 150 software (Li \& Durbin, 2009). Note that this genome is improperly referenced under the name C. intestinalis in several databases because of a recent taxonomic revision (Gissi et al., 2017,

152 and references herein). Between 60 and $67 \%$ of the reads were mapped for $C$. roulei and $C$. intestinalis individuals, and between 83 and $87 \%$ of the reads for $C$. robusta individuals. The

154 aligned RAD data were then processed using the reference mapping pipeline in Stacks v2 set with default parameters (Rochette et al., 2019). Only the SNPs sequenced for at least $80 \%$ of

156 the individuals within a location, present in all the populations and with a maximum 
heterozygosity of $80 \%$ were called using the population function. Additional filtering steps were performed using vcftools (Danecek et al., 2011) in order to remove SNPs with a minor

159 allele count of two or showing significant deviations from Hardy-Weinberg equilibrium (P160 value threshold of 0.05 ) in more than $60 \%$ of the population, with the function 161 filter_hwe_by_pop.pl implemented in dDocent pipeline (Puritz, Hollenbeck, \& Gold, 2014). We 162 additionally removed all polymorphisms private to $C$. robusta populations, as such 163 polymorphisms are neither informative to describe $C$. intestinalis population structure nor to evaluate the extent of introgression across species (which results in shared polymorphisms). At the end of the filtration process, the dataset included 397 individuals (see Table S1 across sampled locations) genotyped at 51,141 SNPs (17,280 with a Minor Allele Frequency (MAF) above 5\%) derived from 5,599 RAD-locus with an average depth across all samples of 59 reads 168 per locus, which was then exported into variant call format (VCF). The VCF was then statistically phased using Beagle v5.2 (Browning \& Browning, 2007) in order to extract haplotypes and conduct phylogenetic analyses as explained below.

Population structure analyses

172 Fine-scale population structure (dataset 1): Because of our interest in identifying 173 introgression in the contact zone, 280 individuals of $C$. intestinalis from 18 sampling sites (70\% 174 of the data) were obtained from the English Channel, Iroise Sea and the Bay of Biscay. We first 175 explored the fine-scale population structure of these populations (dataset 1; Table S1; Figure 1B). The dataset 1 was further filtered to keep SNPs with a MAF above 5\%, and additionally

177 thinned by keeping one random SNP over bin of $1 \mathrm{~kb}$ to take into account physical linkage, 178 using vcftools (Danecek et al., 2011). In total, 13,603 linked SNPs and 3,510 unlinked SNPs 179 were used to study the fine-scale population structure. For the unlinked SNPs, we used the 
180 function find.clusters in adegenet (Jombart, Devillard, \& Balloux, 2010) to find the best 181 number of clusters (lower BIC value) describing the population structure on the 50 first 182 principal components of a PCA (Figure S1). These clusters were then used as discriminant 183 factors to compute a discriminant analysis of principal components (DAPC) with two 184 discriminant functions. We then used the snmf function of the R package LEA (Frichot \& 185 François, 2015), using the number of cluster inferred from the find.clusters function in 186 adegenet to examine the admixture proportions within each location. We estimated pairwise $187 F_{\text {ST }}$ values with $95 \%$ confidence interval among sampling sites by bootstrapping $(10,000$ 188 replications) using the $\mathrm{R}$ package StAMPP (Pembleton, Cogan, \& Froster, 2013) and 189 significance was tested after accounting for multiple testing with Bonferroni correction. The 190 linked SNPs were used to compute another DAPC using the group inferred by the function 191 find.clusters on the unlinked SNPs, extract the eigenvalue of each individual SNP, and evaluate 192 the genomic distribution of the markers responsible for the population structure.

193 Large-scale population structure (dataset 2): For the large-scale population structure 194 analyses, and to achieve a more balanced sampling structure, we kept only one sampling 195 location per genetic cluster inferred with the fine-scale analyses (see above). In total, 129 196 individuals from nine sampling sites for C. intestinalis, 32 individuals from two sampling sites 197 for C. robusta and 19 individuals from one sampling site for $C$. roulei were included (dataset 2; 198 Table S1; Figure 2A). This second dataset was filtered to keep SNPs with a MAF above 5\%, and 199 further filtered for physical linkage (one random SNP per kb). Overall, 17,138 linked and 3,828 200 unlinked SNPs were used depending on the analysis. Using the unlinked SNPs, we conducted 201 a PCA analysis from the R package adegenet (Jombart \& Ahmed, 2011) and admixture analyses 202 (detailed on Figure S2) from the R package LEA (Frichot \& François, 2015). We computed the 
203 pairwise $F_{\text {ST }}$ value with $95 \%$ confidence interval from bootstrapping (10,000 replications) using 204 the R package StAMPP (Pembleton et al., 2013), and significance was tested after accounting 205 for multiple testing using Bonferroni correction. A second PCA analysis was performed on the 206 linked SNPs and the eigenvalue of each SNPs were extracted and plotted against their physical 207 position on the reference genome in order to characterize the genomic distribution of the 208 markers responsible for the population structure. Finally, we used the statistically phased VCF 209 file to create a pseudo sequence of all SNPs, and transformed it into a fasta file containing two 210 haplotypes per individual using a custom R script available in the Zenodo archive (see the Data 211 Accessibility section). From this fasta file, we computed the pairwise genetic distance between 212 each haplotype, and represented a neighbor joining tree based on the GTR substitution model 213 using the R package phangorn (Schliep, 2011).

214 Investigating introgression between Ciona intestinalis and C. robusta (dataset 3): To assess

215 the variability of introgression along the genome, we first used the R package LEA using $k=2$ 216 to compute the individual ancestry of $C$. robusta and $C$. intestinalis individuals, over the whole 217 genome, and for each chromosome independently. Pairwise $F_{S T}$ values between the two 218 sampling locations (pooled) of $C$. robusta and each other locations ( $C$. intestinalis) were then 219 computed for each SNP following the method of Weir and Cockerham (1986) using vcftools 220 (Danecek et al., 2011). For each chromosome, we calculated the maximum $F_{S T}$ value taken 221 over a sliding window of $100 \mathrm{~kb}$, which was then smoothed with the R package ggplot2 222 (Wickham, 2011). Additionally, we created an ancestry informative set of SNPs by extracting 223 the markers differentially fixed (i.e., $F_{S T}$ value of one) between $C$. robusta and the Gul 224 population $(6,849$ SNPs in total). The later population comes from a region and an 
225 environment (deep natural habitat) where $C$. robusta have never been reported, thus, is the 226 least likely population to have recently hybridized with C. robusta.

To detect potential introgression tracts of $C$. robusta within the genome of $C$.

228 intestinalis, we extracted the $F_{\text {ST }}$ value of the 6,849 ancestry informative SNPs calculated

229 between C. robusta and each 22 C. intestinalis populations (Rek excluded due to low sampling

230 size). We then used the Hidden Markov Model (HMM) developed by Hofer, Foll \& Excoffier

231 (2012) to infer the position of genomic islands. Briefly, the HMM characterizes and sorts

232 genomic regions according to their level of differentiation, and is generally used to detect

233 island of divergence (e.g. Soria-Carrasco et al., 2014, Shi et al., 2021). Here, the HMM was

234 applied to detect regions of introgression by contrasting regions with high-background

235 differentiation (with an $F_{\text {ST }}$ value of one) from regions with intermediate differentiation (with

$236 F_{\mathrm{ST}}$ normally distributed around the $15 \%$ lower quantile) and low differentiation (with $F_{\mathrm{ST}}$

237 normally distributed around the $5 \%$ lower quantile). The HMM was run using a modify version

238 of the R script by Marques et al. (2016). To avoid any biases by comparing introgressed

239 population from non-introgressed populations, the quantiles were extracted from the

240 distribution of all $F_{S T}$ values calculated for the 22 pairwise comparisons. Regions of low

241 differentiation covering more than 4 consecutive SNPs, and regions of intermediate

242 differentiation covering more than 10 consecutive SNPs in a given pair of $C$. robusta and $C$.

243 intestinalis were considered as candidate for introgression. Finally, the genotypes of all the

244 individuals at each diagnostic SNP was visualized using a modified version of mk.image of the

245 R package introgress (Gompert \& Buerkle, 2010) developed by Simon et al. (2021). This

246 analysis was performed independently along each chromosome. 


\section{Results}

Fine-scale population structure of Ciona intestinalis in France and UK

Four genetic clusters, which explained $25 \%$ of the variance in the DAPC, were

251 identified in the English Channel, Iroise Sea and Bay of Biscay (Figure 1A). The genetic

252 clustering of the sampling sites was consistent with their geographical proximity, except for

253 three of them grouping with geographically distant ones (Figure 1B): 1) the individuals from

254 Jer (Jersey), geographically close to the northern Brittany populations (deep pink cluster) but

255 clustered with eastern UK sites (blue cluster), 2) the individuals from Rch (La Rochelle) and Bri

256 (Brighton), which belong to the northern Brittany cluster, despite being geographically closer

257 to Western France (yellow cluster) and eastern UK (blue cluster), respectively. This mosaic

258 structure was also supported by the LEA analysis (Figure 1E), notably for Bri and Jer. However,

259 Rch showed evidence of admixture between nearby locations (Qui and Lor in Western

260 Brittany) and distant ones (Per and Blo in Northern Brittany). Additionally, the two

261 westernmost English populations (Ply and Brx) were genetically differentiated from all the

262 other sampling sites (green cluster in Figure 1B, admixture analysis, Figure 1E).

The SNPs contributing to the population structure observed along the first axis

264 were distributed genome-wide (Figure 1C). Conversely, the SNPs structuring the second axis 265 (and thus differentiating the westernmost English populations) were over-represented at the

266 start of two chromosomes (5 and 8, Figure 1D). Pairwise $F_{\text {ST }}$ values were overall low $(0.001<$ $267 F_{\mathrm{ST}}<0.028$ ) but significant across most sites (Table S2), except among four sites of northern 268 and western Brittany ( $\mathrm{AbW}, \mathrm{MBI}, \mathrm{Cam}$, and Lor), and among four sites of the eastern UK (Sth, 
following the coastline, i.e. Rch versus several sampling sites in the UK (Ply, Sho, Poo).

A
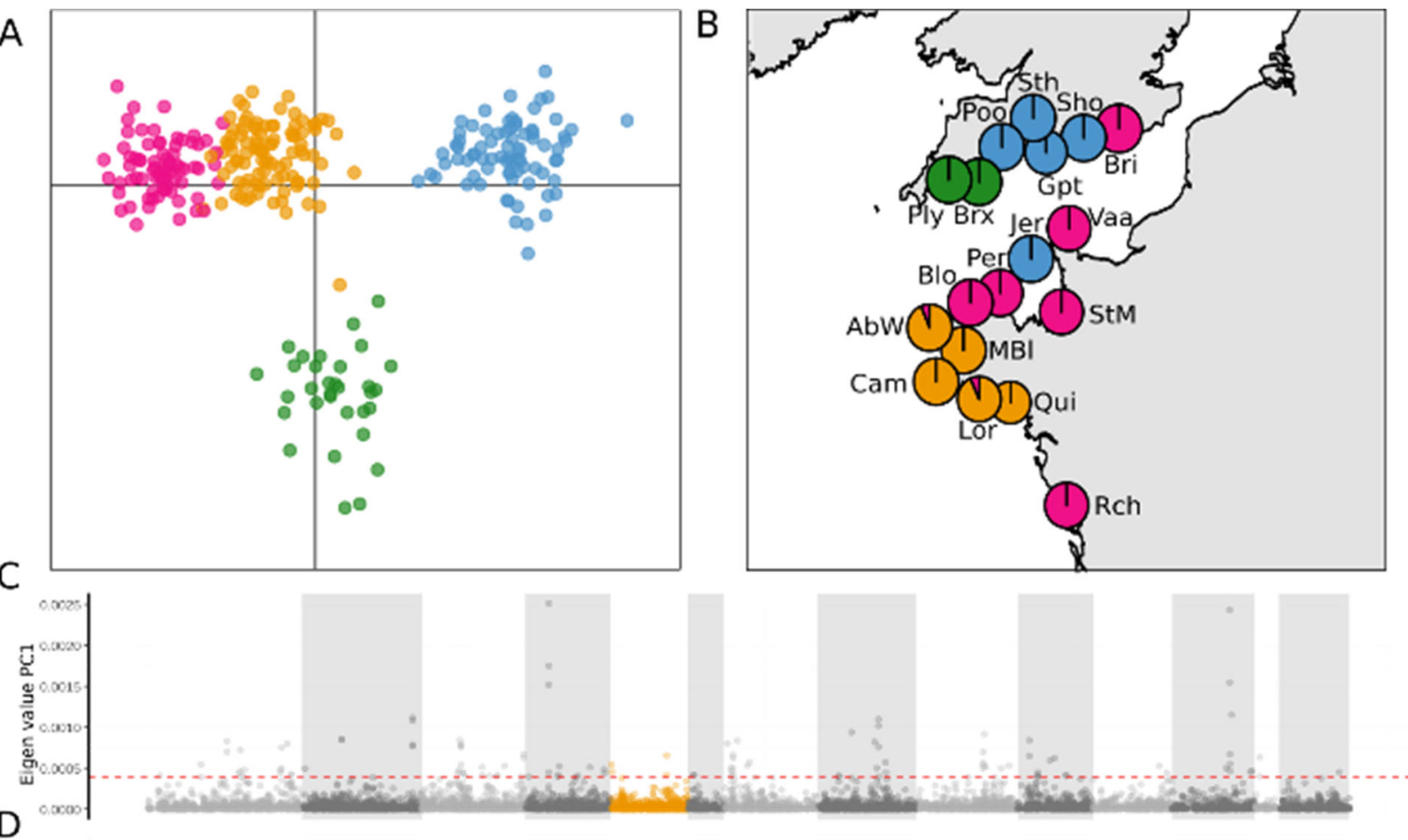

$\mathrm{D}$

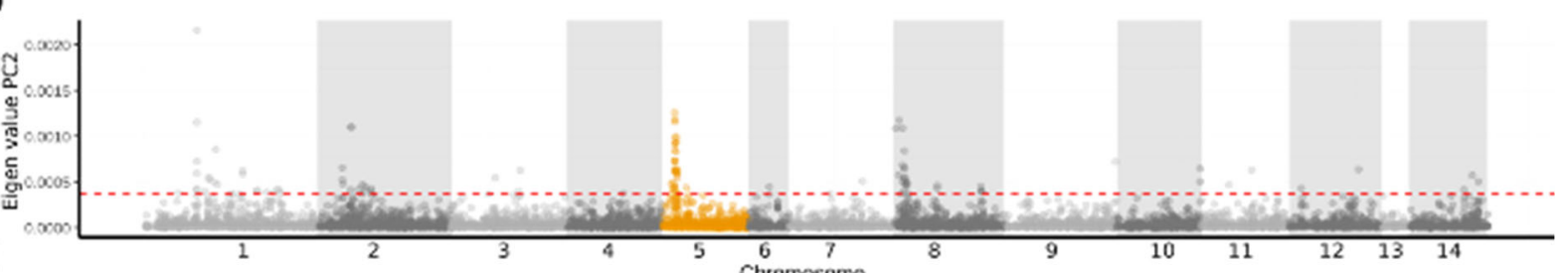

$\mathrm{E}$

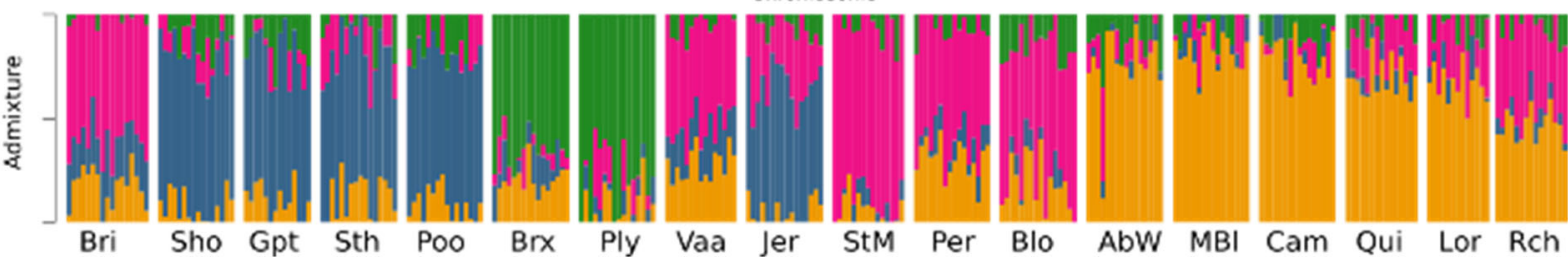

272 Figure 1: Fine-scale structure analysis of the 18 populations of $C$. intestinalis sampled in Bay of Biscay,

273 Iroise Sea and English Channel. Further details about the sampling sites and the code names are 274 provided in Table S1. Figure 1A shows the clustering of 280 individuals genotyped at 3,510 unlinked 275 SNPs with a DAPC set for $K=4$ clusters identified by the find.clusters function in adegenet (Jombart et 276 al. 2010), each cluster is pictured by a different color. The same color scheme is used in the other plots. 277 Figure 1B shows the proportion of individuals per sampling site assigned to each cluster with the DAPC. 278 Figure 1C and 1D display the contribution (eigenvalue) of the 13,603 linked-SNP to the first and second 279 axes of the DAPC, respectively; chromosome 5, which is carrying an introgression hotspot, is 280 highlighted in orange; the dashed red lines show the 95\% quantiles above which are located the top $2815 \%$ eigenvalues. Figure $1 \mathrm{E}$ shows the outcome of an admixture analysis made with the 3,510 unlinked SNPs using the snmf function of the LEA package for K=4 clusters; the ancestry proportion to each cluster is indicated for each individual, which are sorted according to their locations. 
Large-scale population structure across the Northern Atlantic:

The non-indigenous individuals of $C$. robusta are highly divergent from the

287 individuals of the two species native to European waters, C. intestinalis and C. roulei, as 288 illustrated on the first axis of the PCA, which explained $51.80 \%$ of the inertia (Figure 2B). Populations with less genetic divergence are then distinguished in the following axes: the northern European samples of $C$. intestinalis (Gru, Gul and Tro, in blue) are distinguished from the other locations, and from $C$. roulei, by the second axis (2.56\%, figure $2 \mathrm{~B})$. The following axes (3 to 8) distinguished individuals sampled from different locations but with much smaller and decreasing inertia, from $1.65 \%$ to $0.52 \%$ (Figure $2 \mathrm{C}-\mathrm{E}$ ). C. roulei was distinct from $C$. intestinalis only along the axis 5 (1.29\%, Figure 2D). The two sampling sites Rek and Nah, in which $C$. intestinalis has an undetermined status (putatively introduced), were very similar to populations from the English Channel and Bay of Biscay, being distinguished on axes six and eight (Figure 2D,E).

The SNPs contributing to the major divergence between $C$. robusta and the two native species $C$. intestinalis and $C$. roulei were distributed genome-wide (i.e., Figure S3). However, at the start of chromosome 5, a reduction of the divergence between $C$. robusta and $C$. intestinalis was observed, as shown by a slight decline of eigenvalues in the PC1. The population structure depicted by the PCA was corroborated by the admixture analysis (Figure $2 \mathrm{~F}$, Figure S2) showing high support for all the clusters described from the axes one to six of the PCA (Figure 2B-E). All the pairwise $F_{\mathrm{ST}}$ were significantly different from 0 expect for two comparisons (between the two C. robusta sites, and between Rek and Sth, Table S3). The $F_{\text {ST }}$ values ranged from 0.02 to 0.169 among $C$. intestinalis sampling sites, which is similar to the 
308 populations. The two sites Nah and Rek showed the lowest $F_{\mathrm{ST}}$ values with the populations 309 from the English Channel (e.g., $F_{S T}$ Nah vs AbW $=0.033$ and $F_{S T}$ Rek vs Sth non-significant from 310 0), and were less differentiated than pairwise comparisons between northern and southern 311 North Atlantic sites. Very large values (i.e., 0.761 to 0.813 ) were observed between C. robusta 312 and $C$. intestinalis/C. roulei (Table S3). The deep divergence of $C$. robusta from the other 313 populations, and that each population clustered in separate groups, is also confirmed by the 314 phylogenetic tree (Figure 2G). C. roulei individuals formed a distinct group closely related to 315 C. intestinalis. 

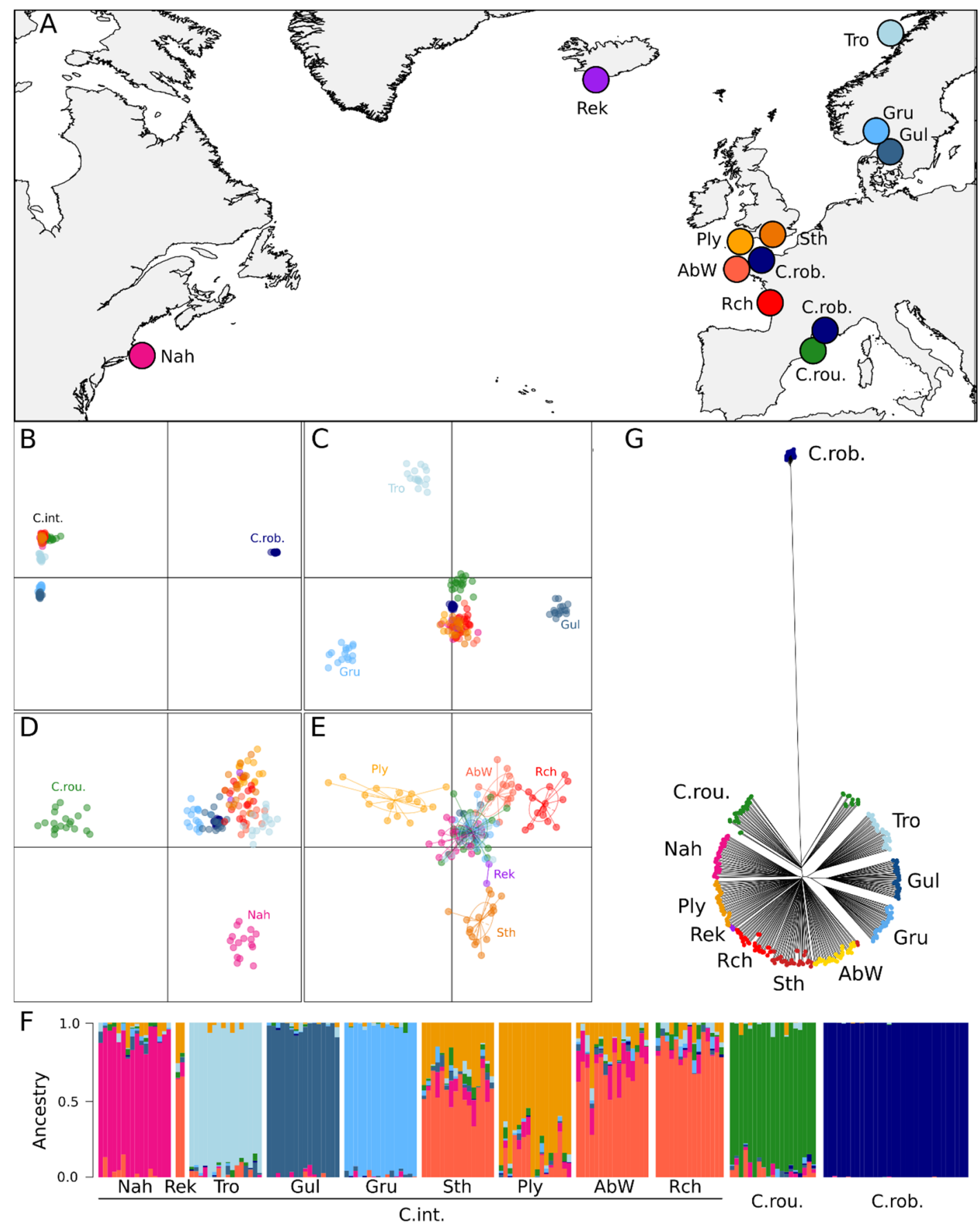

317 Figure 2: Large-scale population structure analysis of Ciona intestinalis (C. int.; native to Europe) populations, $C$. roulei (C.rou.; native to Europe) and C. robusta (C. rob., introduced to Europe). The locations examined are shown in Figure 2A. The location name, and further details, associated to each code are provided in Table S1. The colour code used to picture each sampling location in A) is used in the other plots. PCA plots, based on 180 individuals genotyped at 3,828 unlinked SNPs, are shown in figures $2 \mathrm{~B}$ to $2 \mathrm{E}$, which are displaying 8 different axes (B: 1 vs. 2; C-E: 3 vs. 4,5 vs. 6,7 vs 8 ), associated to $51.80,2.56,1.65,1.45,1.29,0.75,0.55$ and $0.52 \%$ of the total inertia, respectively. Figure $2 \mathrm{~F}$ displays the outcome of the admixture analysis made with the same SNPs using the snmf function of the LEA package for a $\mathrm{k}=8$ clusters (detailed on cluster selection available in Figure S2); the ancestries of each individual are sorted by species and, for $C$. intestinalis, by sampling locations (left part). A neighborjoining tree of 360 phased haplotype built with the 17,138 linked SNPs is pictured in figure 2G. 

genome (Figure S4), with 39.64\% of the SNPs, with a MAF above $5 \%$, being diagnostic using Gul population as reference $\left(F_{\mathrm{ST}}=1\right)$. No sign of genome-wide admixture was detected between the two species (Figure 3A, top panel), but the $C$. roulei individuals appeared admixed with a $C$. robusta ancestry ranging from 1.96 to $8.77 \%$ (Figure 3A, top-panel). The same results were obtained when each chromosome was analyzed independently (Figure S5), with one noticeable exception found on chromosome 5. On this chromosome, 82 C. intestinalis individuals showed a signal of admixture with C. robusta (up 337 to $8.62 \%)$. Chromosome 5 was also the only chromosome where regions of introgression with 338 a low differentiation between $C$. intestinalis and $C$. robusta at diagnostic SNPs were detected by the HMM, all of which being located between 0.61 and $1.58 \mathrm{Mb}$ (tracts sizes ranging from $64.83 \mathrm{~kb}$ to $0.49 \mathrm{Mb}$, Table S4). These regions were found in nine populations, and shared an $80 \mathrm{~kb}$ fragment located from 0.81 to $0.88 \mathrm{Mb}$ of chromosome 5. Large regions of intermediate differentiation, with tracts sizes ranging from $40.04 \mathrm{~kb}$ to $1.25 \mathrm{Mb}$, were also found only on chromosome 5, with $90 \%$ of them located around the region of low differentiation (from 0.40 344 to $2.22 \mathrm{Mb})$. In this portion of chromosome 5, C. robusta ancestry among $C$. intestinalis 345 individuals reached up to $76.77 \%$ (Figure 3A - bottom panel). The presence of admixed individuals were detected at sites located in the contact

347 zone in the English Channel, Iroise Sea and Bay of Biscay (Figure 3B,C, Table S4). In agreement 348 with the HMM analysis, chromosome-wide $F_{\text {ST }}$ values calculated between those populations 349 and $C$. robusta showed a striking decline in $F_{\text {ST }}$ on chromosome 5 (Figure 4). The most extreme 350 drop was localized around $0.87 \mathrm{Mb}$, i.e. within the $80 \mathrm{~kb}$ region of low differentiation shared 
351 among the most introgressed populations. The largest decline in $F_{S T}$ was found in the south 352 western part of the English Channel, in the two populations assigned to the green cluster in 353 the fine-scale analyses (Brx and Ply, Figure 1). Here, $F_{S T}$ decreased below 0.5 (Figure 4 - bottom 354 panel), and 29 out of 32 individuals carried at least one $C$. robusta tract (Figure 3A-B-C). The 355 decline in $F_{\text {ST }}$ was not observed in every locations of the contact zone (Figure 4). For instance, $356 \quad F_{\text {ST }}$ values of one was found throughout the entire chromosome 5 in five out of six comparisons 357 involving sites from the pink cluster identified in the fine-scale analysis (Figure 1), except in 358 Rch (Figure 4 - top panel). In this latter population, a second $F_{\text {ST }}$ decline was visible on the 359 same chromosome around $3.1 \mathrm{Mb}$, which is also a region of intermediate differentiation 360 identified by the HMM analysis (Table S4). The admixture signal, and the long tracts of $C$. 361 robusta ancestry were absent from all the sites outside the contact zone, or from other 362 chromosomes (Figure $3 \mathrm{~A}-\mathrm{B}$, and Table S4), expect in C. roulei where few small tracts of 363 introgression $(<77 \mathrm{~kb})$ were detected on chromosome 7 and 10. 
A

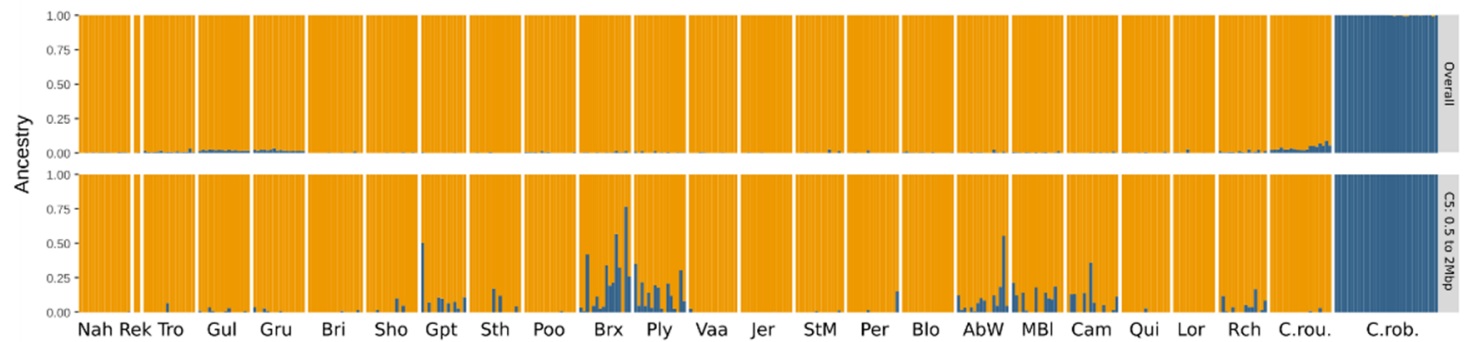

B

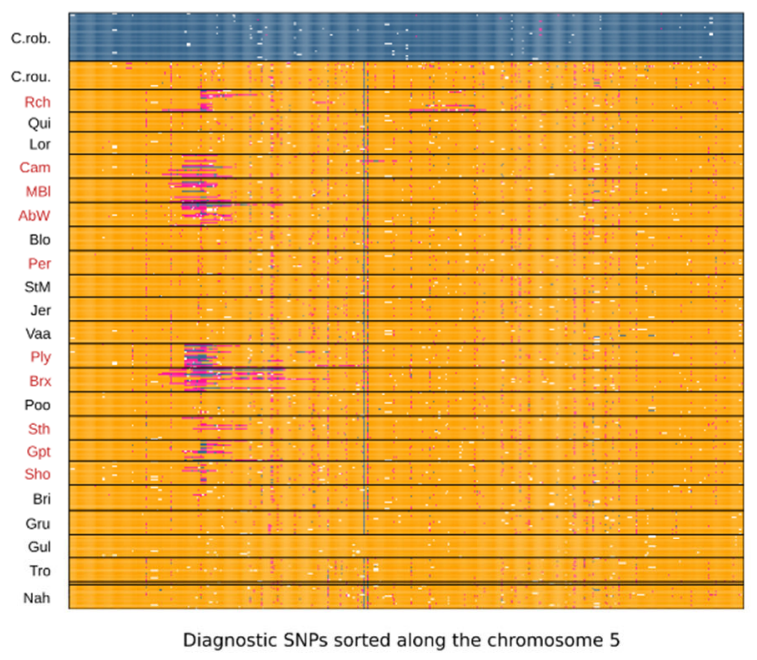

$\mathrm{C}$

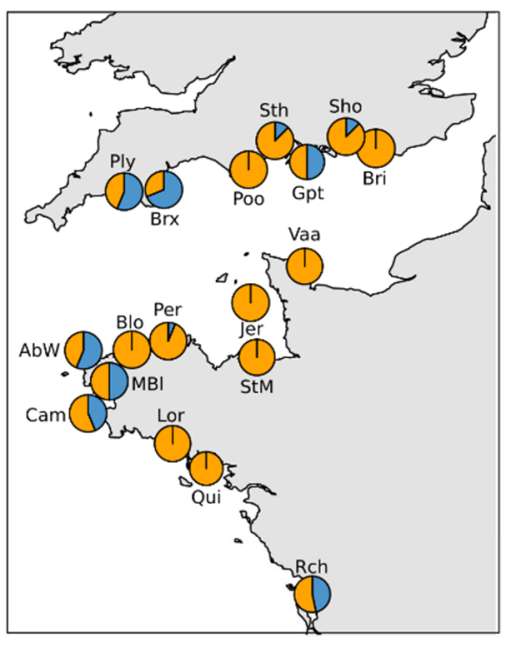

D

Figure 3: Evidence for introgression along chromosome 5 from the introduced species $C$. robusta into the native species $C$. intestinalis. Figure $3 \mathrm{~A}$ shows the admixture plots computed for $\mathrm{K}=2 \mathrm{i}$ ) on 397 individuals genotyped at 17280 linked-SNPs from the overall dataset (top), and ii) on a subset of 354 SNPs located on chromosome 5, from 0.5 to $2.0 \mathrm{Mb}$ (bottom). Figure 3B displays the introgress plot showing the genotypes of the 397 individuals at 545 SNPs chosen to be diagnostic between C. robusta and $C$. intestinalis along chromosome 5 . Individuals ( $y$-axis) are ordered from top to bottom per species (C. robusta: C. rob., C. roulei: C. rou., C. intestinalis); $C$. intestinalis individuals are sorted by location. Populations codes where introgression was detected by the HMM are colored in red. Dark blue boxes indicate homozygote genotype on C. robusta alleles; yellow, homozygote genotype on C. intestinalis alleles; pink, heterozygotes; and white boxes, missing values. Figure $3 C$ shows the proportion of individuals per site displaying a $C$. robusta tract (blue) in chromosome 5. Figure 3D and 3E display a neighbor-joining trees build on 794 phased haplotypes showing the similarities between some $C$. intestinalis haplotypes from admixed locations (red dots) and the haplotypes obtained for C. robusta (dark blue dots on the right divergent branch), when using data for chromosome 5 (D) and a zoom from 0.5 to $2.0 \mathrm{Mb}$ of the same chromosome (E); such similarities are not observed for the haplotypes obtained in locations from the contact zone with no introgression (yellow dots), or from other sites located outside the contact zone and from C. roulei (colored according to the color code used in Figure 2). 

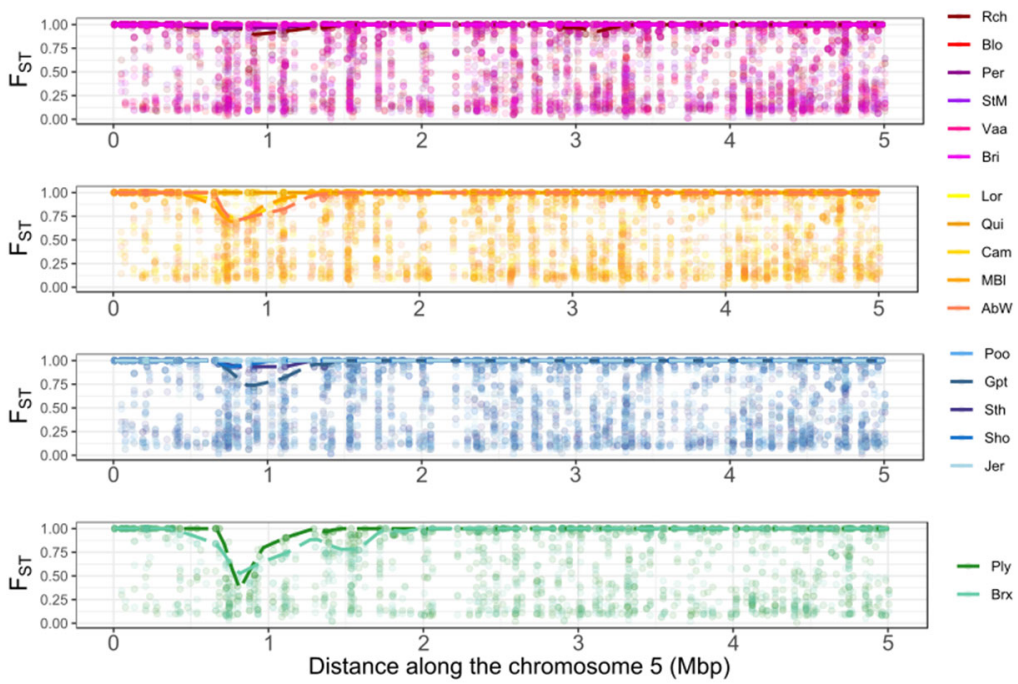

384 Figure 4: Pairwise $F_{\text {ST }}$ values along chromosome 5 between $C$. robusta and populations of $C$. intestinalis 385 sampled in the contact zone between the two species, in the English Channel, Iroise Sea and Bay of 386 Biscay. Each graph corresponds to a comparison made with populations from each of the four genetic 387 clusters identified in the fine-scale analysis (clusters are pictured with the same color code as in Figure 388 1). The population that belongs to each cluster is listed on the right. Each dot represents the $F_{\text {ST }}$ value 389 for the 1235 SNPs with a MAF of $5 \%$ on chromosome 5 , and the dashed lines show the maximum $F_{\text {ST }}$ 390 value computed over bins of $100 \mathrm{~kb}$.

The neighbor-joining tree built with SNPs from chromosome 5 showed that some phased haplotypes from admixed sites (red haplotypes in Figure 3D) were genetically closer to $C$. robusta than the haplotypes from non-admixed sites (yellow haplotypes in Figure 3D). This pattern is exacerbated when zooming on the region spanning from 0.5 to $2.0 \mathrm{Mb}$ (Figure 3E). Interestingly, 23 haplotypes (including 10 from 5 homozygous individuals) completely overlapped with C. robusta haplotypes when focusing on the portion between 0.7 and $1.2 \mathrm{Mb}$ of chromosome 5 (Figure S6B). In this small region, 19 SNPs were species-diagnostic between S6A). Two of these three SNPs might reflect incomplete lineage sorting or parallel mutation. One was indeed polymorphic in several $C$. robusta and one $C$. intestinalis individual from nonadmixed sites (Nah), and the other one was polymorphic in C. robusta and in $10 \mathrm{C}$. intestinalis 
403 sites, two of which are localized outside the contact zone. Conversely, the last of these three

404 SNPs (position 1020271), was polymorphic in C. robusta (MAF = 9\%), with the minor allele only

405 found in three phased haplotypes of the 23 C. intestinalis haplotypes identical to C. robusta

406 haplotypes. The three haplotypes carrying the $C$. robusta minor allele at this specific SNP were

407 all collected from the east of UK (one from Ply and two from Gpt). These three haplotypes,

408 different from other $C$. intestinalis haplotypes, all clustered with other $C$. robusta in the

409 phylogenetic tree (black arrow in Figure S6B). Thus, it seems that different $C$. robusta

410 haplotypes have introgressed admixed $C$. intestinalis populations. In addition, the

411 introgression tracts were variable in size (Figure 3B). This size variation is associated with twin

412 peaks of differentiation among differentially introgressed populations of $C$. intestinalis, as

413 illustrated by pairwise $F_{S T}$ values computed between $\mathrm{Brx}$ and $\mathrm{MBI}$ or $\mathrm{AbW}$ (Figure $5 \mathrm{C}$, below

414 the arrows), and also visible when comparing the sites of Ply and Brx (Figure 5A).

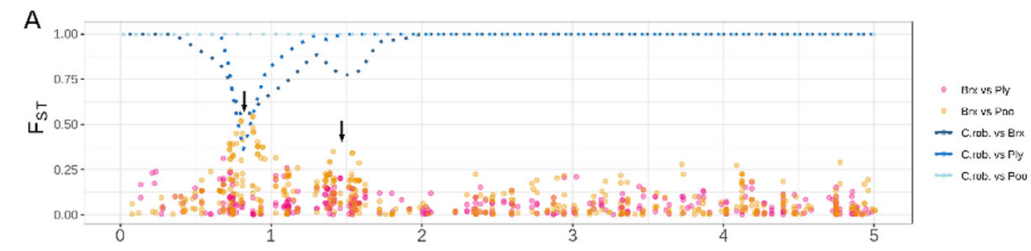

B

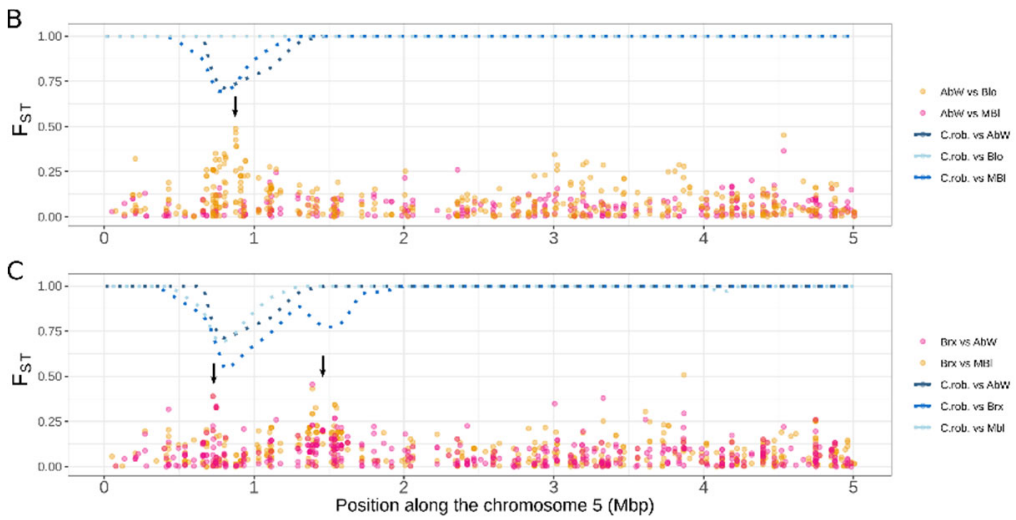

416 Figure 5: $F_{\text {ST }}$ values (orange and pink colors) between pairs of $C$. intestinalis populations and maximum 417 value of $F_{\text {ST }}$ (dashed lines in blue colors) between these $C$. intestinalis populations and $C$. robusta over 418 bins of $100 \mathrm{~kb}$, computed using 1235 SNPs with MAF of 5\% located along chromosome 5 . $F_{\text {ST }}$ is 419 calculated between pairs of sites either A) geographically close in the UK or B) in Brittany (France). In 420 C), $F_{S T}$ is calculated between introgressed sites in France vs. UK. Pink color is used to show $F_{S T}$ between 421 two introgressed sites and orange color between an introgressed and a non introgressed site. 


\section{Discussion}

Using a genome-wide approach based on ddRAD-sequencing, we investigated the

424 fine-scale and large-scale population structure of Ciona intestinalis in native (NE Atlantic) and possibly introduced range (US and Iceland). Our results provide supports to previous studies

426 about the influence of human-mediated transports on population structure at both regional 427 and global scales. Comparisons with its congeners $C$. robusta and $C$. roulei offered new insights 428 about the past and recent history of these three species. Our study finally provided the first evidence of contemporary introgression from C. robusta into $C$. intestinalis, in the introduction range of the former. This introgression was not homogeneously distributed in the genome but rather forms a breakthrough located in an introgression hotspot on chromosome 5.

\section{Chaotic genetic structure and cosmopolitanism: a footprint of human-mediated dispersal}

studies (Bouchemousse et al., 2016a,c; Hudson et al., 2016; Johannesson et al., 2018; Hudson, Johannesson, McQuaid, \& Rius, 2020, Johannesson, Le Moan, Perini, \& André, 2020). In

437 particular, we confirmed that the populations of $C$. intestinalis are highly differentiated over 438 large geographical scales, which is likely due to the presence of different glacial lineages in 439 Europe (Hudson et al., 2020). At smaller geographical scales, $C$. intestinalis is much less 440 genetically structured, and more importantly shows discrepancies between genetic clustering 441 and geographic distance, leading to a mosaic structure. Such a mosaic structure had been 442 previously reported in the study area, with microsatellite markers, and attributed to human443 mediated connectivity among harbors (Hudson et al., 2016). Ciona intestinalis has indeed 444 limited natural dispersal capacity, being characterized by very short-lived larvae $(<24$ hours 
445 under laboratory conditions). Similar mosaic structures have been documented in introduced 446 species inhabiting ports, and characterized by low natural dispersal ability, for example the 447 seaweed Undaria pinnatifida (Guzinski, Ballenghien, Daguin-Thiébaut, Lévêque, \& Viard, 448 2018).

449 The individuals sampled from locations with unclear native vs. non-native status (in 450 the North Western Atlantic and in Iceland) were genetically more similar to the populations 451 sampled in France and England (average $F_{S T}$ of 0.032 ) than to other European populations $\left(F_{S T}\right.$ 452 up to 0.176$)$. They were also more similar among one another than several comparisons within 453 Europe, suggesting that they derived recently from somewhere near the English Channel. A 454 similar situation has been described by Hudson et al. (2020) in a Canadian site located further 455 north than our study population (Nah). However, the Canadian individuals appeared admixed 456 between Swedish and English Channel lineages, while Nah here appeared as a mostly pure 457 cluster genetically close to the English Channel lineage. Population structure along the 458 western Atlantic Coast is common, even in invasive species such as the green crab (Pringle, 459 Blakeslee, Byers, \& Roman, 2011; Jeffery, et al., 2017). The Canadian site is located in Nova 460 Scotia, a major suture zone for marine species living along the Western Atlantic coast, while 461 our sampling site is located further south where populations display usually less admixture 462 (Standley et al., 2018). The different admixture pattern observed here and in Hudson et al. 463 (2020) suggest population structure of $C$. intestinalis in North America that might reflect 464 multiple introduction events from the native range, which is often reported in marine 465 introduced species (Viard, David, \& Darling, 2016). Additional sampling sites along the 466 Western Atlantic coast are needed to further explore the population structure of $C$. intestinalis 467 and reconstruct its introduction history. Our study concurs with previous results by 
468 Bouchemousse et al. (2016a) and Hudson et al. (2020), and suggests that $C$. intestinalis can be described as a neo-cosmopolitan species according to the terminology by Darling \& Carlton

470 (2018), with a trans-Atlantic distribution due to human introductions, rather than to an 471 eucosmopolitan species with relict populations that took advantage of new available habitats 472 (ports and marinas).

474 A continued and needed appraisal of species status within the Ciona genus

The many Ciona species belong to a complex genus, as shown by the recent 476 discovery of a new species in the Mediterranean Sea (i.e., Ciona intermedia, Mastrototaro et 477 al., 2020), and the recent in-depth taxonomic revision of the previously accepted $C$. intestinalis 478 species. Morphological observations (Brunetti et al., 2015), molecular data (Nydam \& 479 Harrison, 2010; Zhan, Macisaac, \& Cristescu, 2010; Bouchemousse et al., 2016a,c) and 480 experimental crosses (Lambert et al., 1990; Malfant et al., 2017) show that two deeply 481 diverged lineages, named type $A$ and type $B$, co-exist under the accepted name $C$. intestinalis. 482 This taxonomic revision restored a previously synonymized species, C. robusta. In line with 483 these previous data, we observed differentially fixed SNPs between $C$. robusta and $C$. 484 intestinalis across most of their genome (average $F_{S T}>0.780$ for all pairwise comparison). This 485 conclusion also holds for $C$. robusta and $C$. roulei. Our results show that the species status of 486 C. roulei needs revision. We showed a weak genome-wide differentiation when comparing $C$. 487 roulei with $C$. intestinalis populations sampled in the English Channel $\left(F_{S T}<0.075\right)$. 488 Interestingly, the differentiation is even weaker than those observed between the southern 489 and northern European populations of $C$. intestinalis studied here ( $F_{\text {ST }}$ up to 0.169). Lambert 490 et al. (1990) and Malfant et al. (2018) performed experimental crosses showing that ' $C$. 
491 intestinalis and $C$. roulei can be hybridized with no signs of outbreeding depression. In 492 addition, mitochondrial sequencing data shows that the two taxa display similar haplotypes 493 (Malfant et al., 2018). Our results agree with these previous studies suggesting that $C$. roulei 494 is a divergent lineage of $C$. intestinalis, likely trapped in cold waters of the northern 495 Mediterranean Sea after post-glacial warming, like other cold-adapted marine species 496 (Platichthys flesus: Borsa, Blanquer, \& Berrebi, 1997; Sprattus sprattus: Debes, Zachos, Hanel, 497 2008; Sagitta setosa: Peijnenburg, Fauvelot, Breeuwer, \& Menken, 2006).

\section{Evidence for contemporary introgression of the native species by its introduced congener}

Our analyses provided several novel results. We observed one peak of intra-specific differentiation on chromosome 5 that corresponded to a decline of inter-specific 502 differentiation between sympatric populations of $C$. robusta and $C$. intestinalis. This decline was only found in a subset of the populations located in the contact zone between the two species, pointing toward introgression from C. robusta into C. intestinalis populations. Introgression was confirmed by the presence of long $C$. robusta ancestry tracts in some $C$. 506 intestinalis individuals sampled across the English Channel and Iroise Sea, and in the Rch site 507 in the Bay of Biscay. These long introgression tracts were primarily found on chromosome 5 508 between positions 0.38 to $2.32 \mathrm{Mb}$ (Table S4), which we refer as an introgression hotspot. 509 Other long tracts were found on chromosome 5 outside the main introgression hotspot in 510 three individuals from Rch and one from Cam. These other tracts close to the introgression 511 hotspot could be either due to independent introgression events or, more likely to tracts that 512 hitchhiked with the introgression at the hotspot. This introgression restricted to a single 513 hotspot explains why it was missed in previous studies using fewer ancestry-informative SNPs 
514 (Bouchemousse et al., 2016b,c). Marker density is thus a key to obtain evidence for very 515 localized introgression (Ravinet et al., 2018, Turissini \& Matute, 2017, Stankowski et al., 2020). $516 \quad$ C. roulei did not show long tracts of $C$. robusta ancestry in chromosome 5, but showed 517 two small tracks $(<78 \mathrm{~kb})$ on chromosome 7 and 10 , and signs of admixture with $C$. robusta 518 were spread across all chromosomes. This admixture pattern and the absence of long 519 haplotypes from $C$. robusta into $C$. roulei suggests that introgression between $C$. roulei and $C$. 520 robusta is ancient. Previous studies examining gene flow between $C$. intestinalis and C. robusta 521 have interpreted the patterns of allele sharing as a consequence of historical rather than 522 recent introgression (Bouchemousse et al., 2016c). The $C$. roulei samples examined here could 523 thus be another example of historical introgression. Alternatively, such introgression could 524 involve another species closely related to C. robusta, which was present in the Mediterranean

525 Sea. Out of the 14 species currently accepted in the genus Ciona (Word Register of Marine 526 Species; http://marinespecies.org/), three other species not included in this study have been 527 reported in the Mediterranean Sea (C. intermedia, Ciona sp. C and Ciona sp. D). Based on 528 mitochondrial phylogeny, Ciona sp. C appears genetically close to C. robusta (Mastrototaro et 529 al., 2020). Thus, carrying out a genome-wide analysis on the Ciona taxa found in the 530 Mediterranean Sea is needed to confirm that the signs of admixture into $C$. roulei are truly 531 due to introgression with C. robusta.

533 Spatial and temporal dynamics of the introgression tracts

The recent invasion of $C$. robusta into the English Channel (Nydam \& Harrison, 535 2010), the absence of large introgression tracts outside of the contact zone including in pop536 ulations outside European Seas (Rek and Nah), the large size of the introgression tracts (>0.5 
$\mathrm{Mb}$ ) and the genetic similarity between introgressed tracts in $C$. intestinalis and $C$. robusta

538 haplotypes (Figure 3), all point toward a recent introgression event (i.e., post-dating the intro-

539 duction of $C$. robusta in Europe in the late $20^{\text {th }}$ or early $21^{\text {st }}$ centuries). Dating the age of an

540 introgression event is not easy when using reduced representation DNA sequencing, such as

541 ddRAD-sequencing. Shchur et al. (2020) provided a theoretical framework for interpreting the

542 timing of introgression, based on the distribution of genomic admixture tract lengths, and in-

543 cluding positive selection effects. However, the underlying assumption of their method may

544 not hold in our case. For example, the level of genome-wide introgression should be suffi-

545 ciently high to estimate the baseline neutral introgression rate, while in our case it is around

$5460.1 \%$ on average (Fraisse et al., unpublished results), with few tracts outside of the chromo-

547 some 5 hotspot. In addition, these methods assume a single pulse of admixture has occurred

548 while multiple admixture events have likely happened in C. intestinalis, as discussed below.

The localized pattern of long (0.84-1.24 Mb, Table S4), and thus likely young, tracts

550 of introgression in a single region of chromosome 5 , is difficult to explain without invoking

551 some sort of selection. Although a localized desert of genes associated with low

552 recombination rates can potentially produce this pattern, such regions usually exist at several

553 places of a genome. In addition, 21 genes are located in the $80 \mathrm{~kb}$ tract shared by the most

554 introgressed populations, from 0.81 to $0.88 \mathrm{Mb}$ of chromosome 5 (listed in Table S5), which

555 does not support the gene desert hypothesis. Moreover, the high variation of $C$. robusta tracts

556 length in $C$. intestinalis suggests recombination is operating efficiently on the introgression

557 hotspot. In addition, the introgression is not fixed in any of the study populations. Simple

558 positive selection (adaptive introgression sensu stricto) also seems unlikely to explain 
synchronous incomplete sweeps at many distant locations belonging to different genetic clusters. Alternative selective scenarios therefore need to invoke other kind of selection such

561 as balancing selection, frequency dependence or heterosis. For instance, as the two species 562 studied here are highly divergent (12\%, which translates into roughly 2,000 non-synonymous 563 substitutions per $\mathrm{Mb}$ ), the introgressed tracts may carry many mutations affecting fitness.

564 Theory predicts an intrinsic benefit of heterozygosity and a cost of admixture (Schneemann, 565 De Sanctis, Roze, Bierne, \& Welch, 2020). Introgressed tracts might provide a fitness 566 advantage when heterozygous at the start of the introgression, through heterosis effect, but 567 a fitness reduction when frequent and homozygote in a $C$. intestinalis background. 568 Furthermore, the heterosis effect could vanish when long tracts are broken down by 569 recombination (Harris \& Nielsen, 2016). Thus, the dynamic of the introgression might change 570 over time, with some haplotypes being first positively selected, and then counter selected as 571 soon as recombination breaks the introgressed haplotypes into smaller pieces (Leitwein, 572 Duranton, Rougemont, Gagnaire, \& Bernatchez, 2020). Examining changes in introgression 573 frequencies and distribution over time is now required to investigate if selection is truly acting 574 and continues to drive introgressed tracts to high frequency. In addition, genome sequencing 575 will better delineate the heart of the introgression hotspot and identify candidate genes on 576 the basis of their function. The length of introgression tracts can be informative about where the contact 578 happened, as it is inversely correlated to the distance from where the introgression first 579 occurred (Leitwein, Duranton, Rougemont, Gagnaire, \& Bernatchez et al., 2020). This effect 580 was documented between the Atlantic and the Mediterranean lineages of European sea bass, 581 Dicentrarchus labrax, where the size of the Atlantic introgression tracts into the 
Mediterranean population is proportionally reduced with the distance from the contact zone

\section{3} between the two lineages, at the Almeria-Oran front (Duranton, Bonhomme, \& Gagnaire, 584 2019). Building on these observations, the length of C. robusta haplotypes in the genome of C. intestinalis individuals was generally larger in western UK (1.24 Mb in Brx, Table S4) than in other populations (e.g., $0.5 \mathrm{Mb}$ in $\mathrm{Gpt} / \mathrm{Sth} / \mathrm{MBI}$ ), suggesting that the introgression occurred there first. Interestingly, introgression was not found restricted to a particular area and shows a chaotic spatial structure similar to what was observed in Hudson et al. (2016), and genome wide in our study. Intra-specific gene flow promoted by human-mediated transport, as previously suggested (Hudson et al., 2016), could explain the chaotic dispersal of introgression tracts. In this context, introgression tracts could be a relevant tool to identify recent humanmediated migration routes (Gagnaire et al., 2015). For instance, while the population Jer 593 seems genetically related to distant populations located in central-eastern UK at the genome594 wide level (Figure 2B), this population shares a lack of introgression tracts with StM (Figure $5953 \mathrm{C})$, a population in close vicinity, and strongly connected through leisure boating. Another non mutually exclusive hypothesis might explain both the mosaic structure of 597 introgression and the variable size of introgression tracts: independent introgression events 598 occurring several times in different populations. For instance, the honey bee (Apis mellifera scutellata) shows repeated introgressions from the African lineage into the European lineage 600 at one genomic location of chromosome 1 in two hybrid zones that are localized more than 601 5,000 kilometers apart (Calfee et al., 2020). Repeated adaptive introgression has also been 602 documented at a gene involved in insecticide resistance in the mosquito Anopheles spp. (Weill 603 et al., 2000; Norris et al., 2015) and in cotton bollworm Helicoverpa spp. (Valencia-Montoya 604 et al., 2020). Although Ciona species are much more divergent than any of the examples 
605 presented above, the contact zones between $C$. intestinalis and C. robusta are not restricted 606 to a few locations, as they co-occurred in syntopy in several isolated harbors along coastlines 607 of Great-Britain and France, which increases the possibility of introgression. We showed that 608 one SNP distinguished two haplotypes of C. robusta found within C. intestinalis individuals, 609 suggesting that introgression from C. robusta into $C$. intestinalis could have happened at least 610 twice. This SNP was only carried by long introgressed tracts, and could be a footprint of more 611 recent introgression contributing to the twin peaks of differentiation observed in the 612 surrounding of the main introgression hotspot between populations that carry introgression 613 tracts of variable size (Figure 5).

614 The possibility of repeated introgression events does not rule out the hypothesis of 615 intra-specific diffusion of the introgression facilitated by human activities. Both mechanisms 616 might have jointly contributed to the current distribution of the introgression tracts. Overall, 617 our study shows that anthropogenic hybridization can be effective in promoting gene flow 618 between species even at late stage of speciation, contributing to the population structure 619 described among contemporary populations. Future work should focus on complete genome 620 sequencing, and temporal sampling of introgressed populations. 


\section{Acknowledgements}

622 The authors are grateful to Sarah Bouchemousse and Sabrina Le Cam for providing samples

623 from Iceland and Norway, to Alan Brelsford and Pierre-Alexandre Gagnaire for providing

624 advice on ddRAD-sequencing and/or scripts for RAD-Seq analyses, and to James Reeve for help

625 in editing the last version of the manuscript. The authors are also most grateful to the

626 Biogenouest Genomer core facility for their technical support, and from the Biogenouest

627 ABIMS Platform for softwares and bioinformatics tools set-up, and access to computing

628 resources. This work benefitted from funding of the French National Research Agency (ANR)

629 with regards the ANR Project HYSEA (no. ANR-12-BSV7-0011).

\section{Data Accessibility}

631 Trimmed fastq files are available under the NCBI bioproject "PRJNA759193". The filtered vcf

632 files used for the fine-scale, large-scale, and introgression analyses, respectively, as well as the

633 R scripts for population genetics and HMM analyses, and the custom scripts for phylogenetic

634 analyses are available as zenodo archives (doi: 10.5281/zenodo.5346932).

\section{Authors' contributions}

636 F.V. and N.B. designed the research. F.V. obtained the funding grants. C.D. and C.R. performed

637 the lab-work. C.R. performed the raw-data filtration. A.LM. performed the bio-informatics

638 analyses with advices from N.B. and C.F.. A.LM., N.B., C.F., and F.V. interpreted the data. A.LM.

639 and F.V. wrote the first-draft of the paper, with substantial inputs from N.B. and C.F.. All

640 authors revised and edited the final manuscript. 


\section{References}

Borsa, P., Blanquer, A., \& Berrebi, P. (1997). Genetic structure of the flounders Platichthys flesus and P. stellatus at different geographic scales. Marine Biology, 129, 233-246. doi: 10.1007/s002270050164

Bouchemousse, S., Bishop, J.D.D., \& Viard, F. (2016a). Contrasting global genetic patterns in two biologically similar, widespread and invasive Ciona species (Tunicata, Ascidiacea). Scientific reports, 6, 24875. doi: $10.1038 /$ srep24875

Bouchemousse, S., Lévêque, L., Dubois, G., \& Viard, F. (2016b). Co-occurrence and reproductive synchrony do not ensure hybridization between an alien tunicate and its interfertile native congener. Ecology and Evolution, 30, 69-87. doi: 10.1007/s10682-015-9788-1

Bouchemousse, S., Lévêque, L. \& Viard, F. (2017). Do settlement dynamics influence competitive interactions between an alien tunicate and its native congener? Ecology and Evolution, 7, 200-213. doi: 10.1002/ece3.2655

Bouchemousse, S., Liautard-Haag, C., Bierne, N., \& Viard, F. (2016c). Distinguishing contemporary hybridization from past introgression with postgenomic ancestry-informative SNPs in strongly differentiated Ciona species. Molecular Ecology, 25, 5527-5542. doi: 10.1111/mec.13854

Brelsford, A., Dufresnes, C., \& Perrin, N. (2016). High-density sex-specific linkage maps of a European tree frog (Hyla arborea) identify the sex chromosome without information on offspring sex. Heredity, 116, 177181. doi: $10.1038 /$ hdy. 2015.83

Browning, S. R., \& Browning, B. L. (2007). Rapid and accurate haplotype phasing and missing-data inference for whole-genome association studies by use of localized haplotype clustering. American Journal of Human Genetics, 81, 1084-1097. doi: 10.1086/521987

Brunetti, R., Gissi, C., Pennati, R., Caicci, F., Gasparini, F., \& Manni, L. (2015). Morphological evidence that the molecularly determined Ciona intestinalis type A and type B are different species: Ciona robusta and Ciona intestinalis. Journal of Zoological Systematics and Evolutionary Research, 53, 186-193. doi: $10.1111 /$ jzs.12101

Calfee, E., Agra, M.N., Palacio, M.A., Ramírez, S.R., \& Coop, G. (2020). Selection and hybridization shaped the rapid spread of African honey bee ancestry in the Americas. PLoS Genetics, 16, e1009038. doi: 10.1371/journal.pgen.1009038

Danecek, P., Auton, A., Abecasis, G., Albers, C.A., Banks, E., DePristo, M.A., ... 1000 Genomes Project Analysis Group (2011). The variant call format and VCFtools. Bioinformatics, 27, 2156-2158. doi: 10.1093/bioinformatics/btr330

Darling, J. A., \& Carlton, J. T. (2018). A framework for understanding marine cosmopolitanism in the Anthropocene. Frontiers in Marine Science, 5, 293. doi: 10.3389/fmars.2018.00293

Debes P.V., Zachos F.E., \& Hanel, R. (2008). Mitochondrial phylogeography of the European sprat (Sprattus sprattus L., Clupeidae) reveals isolated climatically vulnerable populations in the Mediterranean Sea and range expansion in the northeast Atlantic. Molecular Ecology, 17, 3873-88. doi: 10.1111/j.1365294X.2008.03872.x.

Dehal, P., Satou, Y., Campbell, R.K., Chapman, J., Degnan, B., De Tomaso, A., ... Rokhsar, D.S. (2002). The draft genome of Ciona intestinalis: insights into chordate and vertebrate origins. Science, 298, 2157-67. doi: 10.1126/science. 1080049

Duranton, M., Bonhomme, F. \& Gagnaire, P.-A. (2019). The spatial scale of dispersal revealed by admixture tracts. Evolutionary applications, 12, 1743-1756. doi: 10.1111/eva.12829

Faust, E., Halvorsen, K. T., Andersen, P., Knutsen, H., \& André, C. (2018). Cleaner fish escape salmon farms and hybridize with local wrasse populations. Royal Society Open Science, 5, 171752 . doi : 10.1098/rsos.171752

Firth, L. B., Knights, A. M., Bridger, D., Evans, A., Mieskowska, N., Moore, P. J., . . . Hawkins, S. J. (2016). Ocean sprawl: challenges and opportunities for biodiversity management in a changing world. Oceanography and Marine Biology: An Annual Review, 54, 193-269. doi:10.1201/9781315368597

Frichot, E., \& François, O. (2015). LEA: An R package for landscape and ecological association studies. Methods in Ecology and Evolution, 6, 925-929. doi: 10.1111/2041-210X.12382

Gagnaire, P. A., Broquet, T., Aurelle, D., Viard, F., Souissi, A., Bonhomme, F., ... Bierne, N. (2015). Using neutral, selected, and hitchhiker loci to assess connectivity of marine populations in the genomic era. Evolutionary Applications, 8, 769-786. doi: 10.1111/eva.12288 
Gissi, C., Hastings, K.E.M., Gasparini, F., Stach, T., Pennati, R., \& Manni, L. (2017). An unprecedented taxonomic revision of a model organism: the paradigmatic case of Ciona robusta and Ciona intestinalis. Zoologica Scripta, 46, 521-522. doi: 10.1111/zsc.12233

Gompert, Z., \& Alex Buerkle, C. (2010). introgress: a software package for mapping components of isolation in hybrids. Molecular Ecology Resources, 10, 378-384. doi: 10.1111/j.1755-0998.2009.02733.x

Grabenstein, K.C., \& Taylor, S.A. (2018). Breaking barriers: causes, consequences, and experimental utility of human-mediated hybridization. Trends in Ecology \& Evolution, 33, 198-212. doi: 10.1016/j.tree.2017.12.008

Guzinski, J., Ballenghien, M., Daguin-Thiébaut, C., Lévêque, L., \& Viard, F. (2018). Population genomics of the introduced and cultivated Pacific kelp Undaria pinnatifida: Marinas-not farms-drive regional connectivity and establishment in natural rocky reefs. Evolutionary Applications, 11, 1582-1597. doi: 10.1111/eva.12647

Harris, K., \& Nielsen, R. (2016). The genetic cost of Neanderthal introgression. Genetics, 203(2), 881-891. doi: 10.1534/genetics.116.186890

Hofer, T., Foll, M., \& Excoffier, L. (2012). Evolutionary forces shaping genomic islands of population differentiation in humans. BMC genomics, 13, 107. https://doi.org/10.1186/1471-2164-13-107

Hudson, J., Johannesson, K., McQuaid, C.D., \& Rius, M. (2020). Secondary contacts and genetic admixture shape colonization by an amphiatlantic epibenthic invertebrate. Evolutionary Applications, 13, 600-612. doi: 10.1111/eva.12893

Hudson, J., Viard, F., Roby, C., \& Rius, M. (2016). Anthropogenic transport of species across native ranges: unpredictable genetic and evolutionary consequences. Biology Letters, 12, 20160620. doi: $10.1098 / \mathrm{rsbl} .2016 .0620$

Hufbauer, R. A., Facon, B., Ravigné, V., Turgeon, J., Foucaud, J., Lee, C. E., Rey, O., \& Estoup, A. (2012). Anthropogenically induced adaptation to invade (AIAI): contemporary adaptation to human-altered habitats within the native range can promote invasions. Evolutionary Applications, 5, 89-101. doi: 10.1111/j.1752-4571.2011.00211.x

Jeffery, N.W., DiBacco, C., Wringe, B.F., Stanley, R.R.E., Hamilton, L.C., Ravindran, P.N. \& Bradbury, I.R. (2017). Genomic evidence of hybridization between two independent invasions of European green crab (Carcinus maenas) in the Northwest Atlantic. Heredity, 119, 154-165. doi: 10.1038/hdy.2017.22

Johannesson, K., Le Moan, A., Perini, S., \& André, C. (2020). A Darwinian laboratory of multiple contact zones. Trends in Ecology \& Evolution, 35, 1021-1036. doi: 10.1016/j.tree.2020.07.015

Johannesson, K., Ring, A. K., Johannesson, K. B., Renborg, E., Jonsson, P. R., \& Havenhand, J. N. (2018). Oceanographic barriers to gene flow promote genetic subdivision of the tunicate Ciona intestinalis in a North Sea archipelago. Marine Biology, 165, 126. doi: 10.1007/s00227-018-3388-x

Jombart, T., \& Ahmed, I. (2011). adegenet 1.3-1: new tools for the analysis of genome-wide SNP data. Bioinformatics, 27, 3070-3071. doi: 10.1093/bioinformatics/btr521

Jombart, T., Devillard, S., \& Balloux, F. (2010). Discriminant analysis of principal components: a new method for the analysis of genetically structured populations. BMC Genetics, 11, 94. doi: 10.1186/1471-2156-11-94

Lambert, C., Lafargue, F., \& Lambert, G. (1990). Preliminary note on the genetic isolation of Ciona species (Ascidiacea, Urochordata). Vie et Milieu, 40, 293-295. doi: hal-03036352

Leitwein, M., Duranton, M., Rougemont, Q., Gagnaire, P. A., \& Bernatchez, L. (2020). Using haplotype information for conservation genomics. Trends in Ecology \& Evolution, 35(3), 245-258. doi: 10.1016/j.tree.2019.10.012

Malfant, M., Coudret, J., Le Merdy, R. \& Viard, F. (2017). Effects of temperature and salinity on juveniles of two ascidians, one native and one invasive, and their hybrids. Journal of Experimental Marine Biology and Ecology, 497, 180-187. doi: 10.1016/j.jembe.2017.09.019

Malfant, M., Darras, S., \& Viard, F. (2018). Coupling molecular data and experimental crosses sheds light about species delineation: a case study with the genus Ciona. Scientific Reports, 8, 1480. doi: 10.1038/s41598018-19811-2

Mastrototaro, F., Montesanto, F., Salonna, M., Viard, F., Chimienti, G., Trainito, E., \& Gissi, C. (2020). An integrative taxonomic framework for the study of the genus Ciona (Ascidiacea) and description of a new species, Ciona intermedia. Zoological Journal of the Linnean Society, 190, 1193-1216. doi: 10.1093/zoolinnean/zlaa042 
Marques, D. A., Lucek, K., Haesler, M. P., Feller, A. F., Meier, J. I., Wagner, C. E., Excoffier, L., \& Seehausen, O. (2017). Genomic landscape of early ecological speciation initiated by selection on nuptial colour. Molecular ecology, 26(1), 7-24. https://doi.org/10.1111/mec.13774

Turissini, D. A., \& Matute, D. R. (2017). Fine scale mapping of genomic introgressions within the Drosophila yakuba clade. PLoS Genetics, 13, e1006971. doi: 10.1371/journal.pgen.1006971

McFarlane, S. E., \& Pemberton, J. M. (2019). Detecting the true extent of introgression during anthropogenic hybridization. Trends in Ecology \& Evolution, 34, 315-326. doi: 10.1016/j.tree.2018.12.013

Norris, L. C., Main, B. J., Lee, Y., Collier, T. C., Fofana, A., Cornel, A. J., \& Lanzaro, G. C. (2015). Adaptive introgression in an African malaria mosquito coincident with the increased usage of insecticide-treated bed nets. Proceedings of the National Academy of Sciences of the United States of America, 112, 815820. doi: 10.1073/pnas.1418892112

Nydam, M. L., \& Harrison, R. G. (2010). Polymorphism and divergence within the ascidian genus Ciona. Molecular Phylogenetics and Evolution, 56, 718-726. doi: 10.1016/j.ympev.2010.03.042

Peijnenburg K.T., Fauvelot C., Breeuwer J.A., \& Menken, S.B. (2006). Spatial and temporal genetic structure of the planktonic Sagitta setosa (Chaetognatha) in European seas as revealed by mitochondrial and nuclear DNA markers. Molecular Ecology, 15, 3319-38. doi: 10.1111/j.1365-294X.2006.03002.x.

Pembleton, L. W., Cogan, N. O., \& Forster, J. W. (2013). StAMPP: An R package for calculation of genetic differentiation and structure of mixed-ploidy level populations. Molecular Ecology Eesources, 13, 946952. doi : 10.1111/1755-0998.12129

Popovic, I., Matias, A.M.A., Bierne, N., \& Riginos, C. (2020). Twin introductions by independent invader mussel lineages are both associated with recent admixture with a native congener in Australia. Evolutionary Applications, 13, 515-532. doi: 10.1111/eva.12857

Pringle, J. M., Blakeslee, A. M., Byers, J. E., \& Roman, J. (2011). Asymmetric dispersal allows an upstream region to control population structure throughout a species' range. Proceedings of the National Academy of Sciences of the United States of America, 108, 15288-15293. doi: 10.1073/pnas.1100473108

Puritz, J. B., Hollenbeck, C. M., \& Gold, J. R. (2014). dDocent: a RADseq, variant-calling pipeline designed for population genomics of non-model organisms. PeerJ, 2, e431. https://doi.org/10.7717/peerj.431

Ravinet, M., Yoshida, K., Shigenobu, S., Toyoda, A., Fujiyama, A., \& Kitano, J. (2018). The genomic landscape at a late stage of stickleback speciation: High genomic divergence interspersed by small localized regions of introgression. PLoS Genetics, 14, e1007358. doi : journal.pgen.1007358

Rochette, N.C., Rivera-Colón, A.G., \& Catchen, J.M. (2019). Stacks 2: Analytical methods for paired-end sequencing improve RADseq-based population genomics. Molecular Ecology, 28, 4737-4754. doi: 10.1111/mec.15253

Roux, C., Fraïsse, C., Romiguier, J., Anciaux, Y., Galtier, N., \& Bierne, N. (2016). Shedding light on the grey zone of speciation along a continuum of genomic divergence. PLOS Biology, 14, e2000234. doi: 10.1371/journal.pbio.2000234

Roux, C., Tsagkogeorga, G., Bierne, N., \& Galtier, N. (2013). Crossing the species barrier: genomic hotspots of introgression between two highly divergent Ciona intestinalis species. Molecular Biology and Evolution, 30, 1574-1587. doi: 10.1093/molbev/mst066

Shchur, V., Svedberg, J., Medina, P., Corbett-Detig, R., \& Nielsen, R. (2020). On the Distribution of Tract Lengths During Adaptive Introgression. G3: Genes|Genomes|Genetics, 10(10), 3663-3673. doi:10.1534/g3.120.401616

Schliep, K.P. (2011). phangorn: phylogenetic analysis in R. Bioinformatics, 27, 592-593. doi: 10.1093/bioinformatics/btq706

Schneemann, H., De Sanctis, B., Roze, D., Bierne, N. \& Welch, J.J. (2020). The geometry and genetics of hybridization. Evolution, 74, 2575-2590. doi: 10.1111/evo.14116

Seebens, H., Blackburn, T.M., Dyer, E.E., Genovesi, P., Hulme, P.E., Jeschke, J.M., ... Essl, F. (2017). No saturation in the accumulation of alien species worldwide. Nature Communications, 8, 14435. doi: $10.1038 /$ ncomms 14435

Shi, Y., Bouska, K. L., McKinney, G. J., Dokai, W., Bartels, A., McPhee, M. V., \& Larson, W. A. (2021). Gene flow influences the genomic architecture of local adaptation in six riverine fish species. BioRxiv. doi: 10.1101/2021.05.18.444736

Simon, A., Arbiol, C., Nielsen, E.E., Couteau, J., Sussarellu, R., Burgeot, T., ... Bierne, N. (2020). Replicated anthropogenic hybridizations reveal parallel patterns of admixture in marine mussels. Evolutionary Applications, 13, 575-599. doi: 10.1111/eva.12879 
Simon, A., Fraïsse, C., El Ayari, T., Liautard-Haag, C., Strelkov, P., Welch, J. J., \& Bierne, N. (2021). How do species barriers decay? Concordance and local introgression in mosaic hybrid zones of mussels. Journal of Evolutionary Biology, 34, 208- 223. doi: 10.1111/jeb.13709

Soria-Carrasco, V., Gompert, Z., Comeault, A. A., Farkas, T. E., Parchman, T. L., Johnston, J. S., Buerkle, C. A., Feder, J. L., Bast, J., Schwander, T., Egan, S. P., Crespi, B. J., \& Nosil, P. (2014). Stick insect genomes reveal natural selection's role in parallel speciation. Science, 344(6185), 738-742. doi: 10.1126/science.1252136

Stankowski, S., Westram, A. M., Zagrodzka, Z. B., Eyres, I., Broquet, T., Johannesson, K., \& Butlin, R. K. (2020). The evolution of strong reproductive isolation between sympatric intertidal snails. Philosophical Transactions of the Royal Society of London. Series B, Biological sciences, 375, 20190545. doi: 10.1098/rstb.2019.0545.

Stanley, R., DiBacco, C., Lowen, B., Beiko, R. G., Jeffery, N. W., Van Wyngaarden, ... Bradbury, I. R. (2018). A climate-associated multispecies cryptic cline in the northwest Atlantic. Science Advances, 4, eaaq0929. doi: 10.1126/sciadv.aaq0929

Turissini, D. A., \& Matute, D. R. (2017). Fine scale mapping of genomic introgressions within the Drosophila yakuba clade. PLoS Genetics, 13, e1006971. doi: 10.1371/journal.pgen.1006971

Valencia-Montoya, W. A., Elfekih, S., North, H. L., Meier, J. I., Warren, I. A., Tay, W. T., Gordon, K., Specht, A., Paula-Moraes, S. V., Rane, R., Walsh, T. K., \& Jiggins, C. D. (2020). Adaptive introgression across semipermeable species boundaries between local Helicoverpa zea and invasive Helicoverpa armigera moths. Molecular biology and evolution, 37, 2568-2583. doi: 10.1093/molbev/msaa108

Viard, F., David, P. \& Darling, J. (2016). Marine invasions enter the genomic era: Three lessons from the past, and the way forward. Current Zoology, 62, 629-642. doi: 10.1093/cz/zow053

Viard, F., Riginos, C., \& Bierne, N. (2020). Anthropogenic hybridization at sea: Three evolutionary questions relevant to invasive species management. Philosophical Transactions of the Royal Society of London. Series B, Biological sciences, 375, 20190547. doi: 10.1098/rstb.2019.0547

Weill, M., Chandre, F., Brengues, C., Manguin, S., Akogbeto, M., Pasteur, N., Guillet, P., \& Raymond, M. (2000). The kdr mutation occurs in the Mopti form of Anopheles gambiae s.s. through introgression. Insect Molecular Biology, 9, 451-455. doi: 10.1046/j.1365-2583.2000.00206.x

Weir, B.S., \& Cockerham, C.C. (1984). Estimating F-Statistics for the analysis of population structure. Evolution, 38, 1358-1370. doi: 10.2307/2408641

Wickham, H. (2011). ggplot2. WIREs Computational Statistics, 3, 180-185. doi: 10.1002/wics.147

Zhan, A., Macisaac, H.J., \& Cristescu, M.E. (2010). Invasion genetics of the Ciona intestinalis species complex: from regional endemism to global homogeneity. Molecular Ecology, 19, 4678-4694. doi: 10.1111/j.1365-294X.2010.04837.x 